\title{
Economic impact of treating invasive mold disease with isavuconazole compared with liposomal amphotericin B in the UK
}

\author{
Emma Bagshaw1 ${ }^{1}$, David A Enoch², Michael Blackney¹, Jan Posthumus ${ }^{3}$ \& Daniel Kuessner*,3 \\ ${ }^{1}$ Covance Market Access, London, UK \\ ${ }^{2}$ Clinical Microbiology \& Public Health Laboratory, Public Health England, National Infection Service, Box 236, Addenbrooke's \\ Hospital, Cambridge University Hospitals NHS Foundation Trust, Cambridge, UK \\ ${ }^{3}$ Basilea Pharmaceutica International Ltd, Basel, Switzerland \\ *Author for correspondence: daniel.kuessner@basilea.com
}

\begin{abstract}
Aim: Invasive mold diseases (IMDs) are associated with significant morbidity and mortality. Approved treatments include voriconazole (VORI), liposomal amphotericin B (L-AMB), posaconazole (POSA) and isavuconazole (ISAV). A UK-based economic model was developed to explore the cost of treating IMDs with ISAV versus L-AMB followed by POSA. Materials \& methods: As indirect comparisons have demonstrated similar efficacy between the comparators, a cost-minimization approach was taken. Drug acquisition, administration \& monitoring, and hospitalization costs were evaluated from the healthcare system perspective. Results: Per-patient costs were UK£14,842 with ISAV versus UK $£ 18,612$ with L-AMB followed by POSA. Savings were driven by drug acquisition, and administration \& monitoring costs. Conclusion: ISAV has the potential to reduce IMD treatment costs relative to L-AMB followed by POSA.
\end{abstract}

First draft submitted: 16 April 2018; Accepted for publication: 31 May 2018; Published online: 18 June 2018

Keywords: aspergillosis $\bullet$ cost $\bullet$ economic $\bullet$ healthcare $\bullet$ invasive fungal infection $\bullet$ invasive mold disease $\bullet$ isavuconazole • liposomal amphotericin B • mucormycosis • posaconazole

Invasive mold diseases (IMDs) are severe fungal infections caused by Aspergillus species and other filamentous fungi, such as Mucorales. Although rare - invasive aspergillosis (IA) and mucormycosis affect between 0.14-2 [1,2] and $0.009-0.06[2,3]$ people per 10,000 in Europe each year, respectively - the threat of IMDs is rising as the size of the at-risk immunocompromised and critically ill population increases [2]. IMDs pose a substantial clinical burden to already vulnerable individuals, such as hematology and oncology patients and transplant recipients, complicating the management of their primary condition and potentially jeopardizing outcomes. Additionally, the economic burden of IMD management is substantial. Prolonged antifungal therapy and lengthy hospitalizations result in substantial treatment costs [4,5], while management of adverse events (AEs) can add to total costs and increase the duration of hospital stays [6].

In the UK, voriconazole (VORI) is currently the standard first-line treatment for IMD, as it is efficacious in IA [7] and has relatively low acquisition costs. However, it lacks activity against Mucorales, use of its intravenous (iv.) formulation is restricted in patients with renal impairment, and it is associated with skin, eye and liver AEs, drugdrug interactions, and a risk of cardiac QT prolongation [8]. Consequently, in IMD cases where differential diagnosis between IA and mucormycosis has not been achieved or VORI is otherwise inappropriate, alternative treatments need to be considered. Liposomal amphotericin B (L-AMB) is currently the most commonly used alternative, as it has broad antifungal activity. However, it is more expensive than VORI, and is associated with metabolic disturbances, infusion-related AEs and renal toxicity. It also requires iv. administration, which necessitates a step down to oral posaconazole (POSA) for outpatient maintenance treatment [9]. In addition to maintenance therapy, POSA is sometimes used in mucormycosis treatment as an alternative to L-AMB, or as salvage therapy [10,11]. However, it is not licenced for the treatment of mucormycosis [12] and its clinical value in the treatment of IA is also limited, as it has only a second-line treatment indication. Several other treatments also have some IMD activity, such as the echinocandins (anidulafungin, caspofungin and micafungin), which are active against Aspergillus species

Future $\because$ Medicine 
and some Penicillium strains; however, they lack activity against Mucorales. The only echinocandin licensed in the EU for the treatment of IA is caspofungin, and this is restricted to patients refractory to, or intolerant of, other agents [13], as it has only been evaluated in noncomparative studies [14].

Limited data exist on the proportional usage of each IMD treatment in clinical practice. However, two recent independent studies by Micallef et al. [15] and Ceesay et al. [4], in which L-AMB and VORI were among the most frequently administered antifungal treatments, may represent common practice in the UK, suggesting that, in cases where VORI is inappropriate, L-AMB is a commonly used alternatively.

Isavuconazole (ISAV) is the most recently approved treatment option for IMD (2015, by the European Medicines Agency [16]). It is a triazole antifungal indicated in the EU in adults for the treatment of IA, and for the treatment of mucormycosis in patients for whom amphotericin B (AMB) is inappropriate. In the treatment of IA, ISAV has comparable efficacy to VORI, as established in the Phase III randomized, controlled SECURE study [17]. ISAV has also been shown to have comparable efficacy in IA to L-AMB, demonstrated in a mixed treatment comparison meta-analysis, which included the SECURE study and two randomized controlled trials of L-AMB in proven/probable IA $[18,19]$. In the treatment of mucormycosis, ISAV has been shown to have comparable efficacy to L-AMB, as demonstrated in a case-control analysis between the Phase III, single-arm VITAL study and the FungiScope $^{\top \mathrm{M}}$ registry database [20]. ISAV is also well-tolerated compared with VORI [17]. Unlike VORI, which can extend QT interval, ISAV has a dose-related shortening effect, with no evidence of associated cardiac risk [21]. ISAV is also anticipated to offer safety and tolerability benefits over L-AMB, given L-AMB's well-documented association with renal toxicity [22-25].

To help inform treatment decisions for IMD cases in which VORI is considered unsuitable, and evaluate the related costs of these treatment decisions, an economic model was developed to estimate the per patient cost to the UK National Healthcare Service (NHS) of treating IMD patients using ISAV alone compared with a regimen of L-AMB followed by maintenance POSA (L-AMB $\rightarrow$ POSA).

\section{Materials \& methods}

\section{Model structure \& key assumptions}

A cost-minimization approach was chosen to model the per patient cost of treating adult IMD patients using ISAV compared with L-AMB $\rightarrow$ POSA. As the available evidence indicates that ISAV has comparable efficacy to L-AMB in the treatment of both IA and mucormycosis $[20,26]$ the model assumed no difference in clinical outcomes between treatment arms, and only differences in costs were considered.

Resource use and costs were aligned where possible with the data sources used in the mixed treatment comparison meta-analysis that demonstrated comparable efficacy between ISAV and L-AMB in the treatment of IA [26]. Therefore, ISAV was assumed to be administered as in the SECURE study [17], while L-AMB was administered as reported in a randomized controlled trial by Leenders et al. [18], which was a key study in the meta-analysis.

The model was developed from the UK NHS perspective and evaluated total direct IMD treatment costs per patient. Costs comprised on-treatment drug acquisition, administration \& monitoring and hospitalization costs (Figure 1). The time horizon was until the end of IMD treatment at either resolution of infection or death. Costs beyond this point were not anticipated to differ substantially between treatment arms. Costs were calculated in 2017 UK£, with source values adjusted to the year of reporting where necessary with the use of a UK consumer price index [27].

\section{Model parameters \\ Drug acquisition}

Drug acquisition costs included only those for ISAV, L-AMB and POSA (Table 1). The administration regimens for each treatment are presented in Figure 2. It was assumed that ISAV was dosed according to its summary of product characteristics (SPC): $2 \times 100 \mathrm{mg}$ oral capsules or $1 \times 200 \mathrm{mg}$ vial for iv. infusion per day, with a loading dose of $3 \times 200 \mathrm{mg}$ (iv. or oral) administered on days 1 and 2 [16]. A total of $25 \%$ of ISAV patients were assumed to be initiated on oral therapy, while the remaining $75 \%$ started on iv. therapy before moving to oral therapy according to mean iv. duration in the SECURE study (8.1 days) [17,28]. Intravenous L-AMB was administered at $3 \mathrm{mg} / \mathrm{kg}$ for $50 \%$ of patients and at $5 \mathrm{mg} / \mathrm{kg}$ for the remaining $50 \%$. The dose range selected reflects the doses recommended in L-AMB's SPC for the treatment of general mycoses $(3 \mathrm{mg} / \mathrm{kg})$ and mucormycosis $(5 \mathrm{mg} / \mathrm{kg})$ [20]. L-AMB dosing was applied according to the weight distribution in the SECURE study (mean $68.6 \mathrm{~kg}$, standard deviation $16.4 \mathrm{~kg}$ ) [17]. The cost of any unused vial contents - as L-AMB should not be stored for later use once 
Total cost ISAV

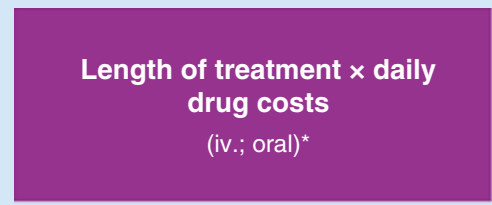

Administration costs

(IV treatment days $\times$ iv. administration cost)

Monitoring costs (tests applied $\mathrm{x}$ test costs)

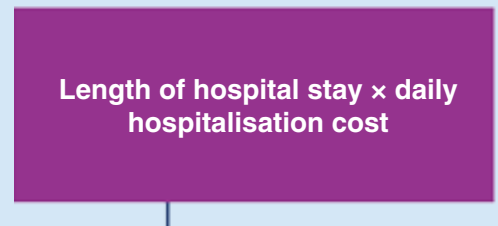

\section{Total cost L-AMB $\rightarrow$ POSA}

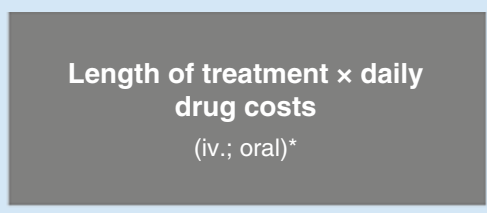

Administration costs

(IV treatment days $\times$ iv. administration cost)

$+$

Monitoring costs

(tests applied $\times$ test costs)

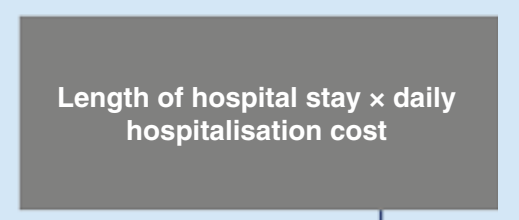

Results of cost-minimisation analysis

(total cost of L-AMB $\rightarrow$ POSA) - (total cost of ISAV)

Figure 1. Model structure.

*Including loading dose as applicable.

ISAV: Isavuconazole; iv.: Intravenous; L-AMB: Liposomal amphotericin B; POSA: Posaconazole.

\begin{tabular}{l}
\begin{tabular}{ll|}
\hline Table 1. Acquisition costs. & \\
\hline Cost factor & Unit cost (UKf) \\
\hline Drugs: & $297.84[29]$ \\
\hline - ISAV (200 mg vial) & $599.28[29]$ \\
\hline - ISAV (14-pack of $100 \mathrm{mg}$ tablets) & $82.19[30]$ \\
\hline - L-AMB (50 mg vial) & $596.96[31]$ \\
\hline - POSA (24-pack of 100 mg tablets) & $0.59[32]$ \\
\hline iv. administration (per minute) & $1.21[33]$ \\
\hline Urinalysis, serum creatinine test, liver function test (per test) & $61.97[34]$ \\
\hline Therapeutic drug monitoring (per test) & $427.23[33]$ \\
\hline Hospital ward (per day) & \\
\hline ISAV: Isavuconazole; iv.: Intravenous; L-AMB: Liposomal amphotericin B; POSA: Posaconazole. \\
\hline
\end{tabular} \\
\hline
\end{tabular}

opened [20] - was included in the calculation of total amount of drug used. All L-AMB $\rightarrow$ POSA patients were assumed to receive iv. L-AMB for the median duration in Leenders et al. (14.5 days) [18], before stepping down to oral POSA for maintenance treatment. POSA was dosed as per its SPC guidance for the treatment of patients with refractory invasive fungal infections/those intolerant to first-line therapy: $3 \times 100 \mathrm{mg}$ oral tablets per day, with a loading dose of $600 \mathrm{mg}(2 \times 300 \mathrm{mg})$ on day 1 [23]. The total treatment duration in both arms was assumed to be equal to the mean total duration of ISAV administered in the SECURE study (47.1 days) [17]. UK list prices for all drugs were taken from the British National Formulary: 


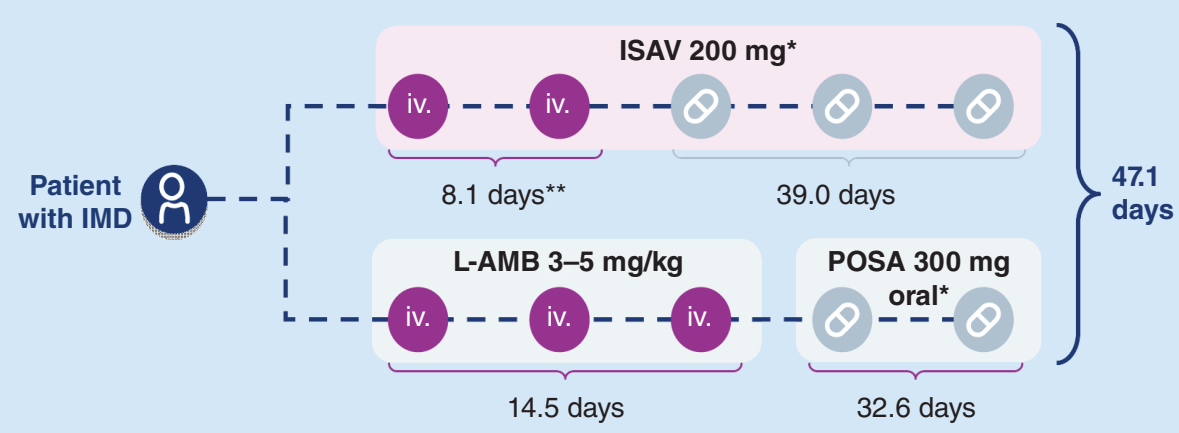

Figure 2. Drug regimens.

*Plus loading doses as described in the text;

**Patients initiated on oral therapy received 0.0 days iv. and 47.1 days oral therapy.

IMD: Invasive mold disease; ISAV: Isavuconazole; iv.: Intravenous; L-AMB: Liposomal amphotericin B; POSA:

Posaconazole.

- ISAV: UK£599.28 per 14-pack of $100 \mathrm{mg}$ capsules and UK£297.84 per $200 \mathrm{mg}$ vial [29].

- L-AMB: UK£82.19 per $50 \mathrm{mg}$ vial [30].

- POSA: UK£596.96 per 24-pack of $100 \mathrm{mg}$ tablets [31].

\section{Administration \& monitoring}

The model included administration costs associated with iv. infusion pack preparation time, and monitoring costs associated with laboratory tests and therapeutic drug monitoring (TDM; Table 1).

Costs of UK£17.95 and UK£5.98 were applied per iv. administration of L-AMB and ISAV, respectively. Based on expert opinion, iv. infusion preparation times of 30 and $10 \mathrm{~min}$, respectively, were multiplied by the cost per working hour of a NHS hospital nurse (Band 5), as reported in the Personal Social Services Research Unit (PSSRU) Unit Costs of Health and Social Care 2016 [32]. Costs were adjusted for inflation.

Laboratory testing was included at UK£1.21 per urinalysis, serum creatinine, magnesium or liver function test, based on the unit price for clinical biochemistry reported in NHS Reference Costs for 2015-2016 [33]. Costs were adjusted for inflation. Based on AEs and precautions listed in SPCs, and expert opinion on testing frequencies, $\mathrm{L}-\mathrm{AMB} \rightarrow$ POSA patients were assumed to require urinalysis, serum creatinine tests and liver-function tests approximately once weekly (seven-times in total), plus six magnesium tests, while ISAV patients were assumed to require seven liver-function tests.

TDM was included at UK£61.97 per sample, based on the price listed by the Mycology Reference Centre, Manchester [34]. TDM was assumed to be required twice over the course of treatment for POSA, based on British Society for Medical Mycology guidelines [35], and once for ISAV.

\section{Hospitalizations}

Hospitalization costs were estimated by multiplying the duration of hospitalization by a daily ward cost (Table 1). The mean duration of hospitalization of all patients in the SECURE study (19.7 days) was used for both ISAV and L-AMB $\rightarrow$ POSA, as no appropriate data were available for the L-AMB $\rightarrow$ POSA regimen [17,28]. Daily ward costs of UK£427.23 were calculated using the weighted average of the unit prices for elective and nonelective excess bed days for Malignant Disorders of Lymphatic or Hematological Systems reported in NHS reference costs for 2015-2016 [33]. Costs were adjusted for inflation. The price for lymphatic and hematological system disorders was used as this patient group was considered representative of those most at risk of IMD. Although many of these patients are treated in intensive therapy units, this was ignored for simplicity. 
Table 2. Results of base case cost-minimization analysis.

\begin{tabular}{|c|c|c|c|c|c|}
\hline \multirow[t]{2}{*}{ Cost source } & \multicolumn{2}{|c|}{ ISAV } & \multicolumn{2}{|c|}{ L-AMB $\rightarrow$ POSA } & \multirow{2}{*}{$\begin{array}{l}(\text { L-AMB } \rightarrow \text { POSA }) \text { - ISAV } \\
\text { Cost difference, UK } £(\%)\end{array}$} \\
\hline & Resource use & Cost, UK $£$ & Resource use & Cost, UK $£$ & \\
\hline iv. drug & 8.1 days $^{\dagger}$ & 2703 & 14.5 days & 7271 & $4568(63)$ \\
\hline Oral drug & 39.0 days $^{\dagger}$ & 3598 & 32.6 days & 2507 & $-1091(-43)$ \\
\hline Total drug acquisition & 47.1 days & 6301 & 47.1 days & 9778 & $3478(36)$ \\
\hline Hospitalization & 19.7 days & 8416 & 19.7 days & 8416 & $0(0)$ \\
\hline $\begin{array}{l}\text { Administration \& } \\
\text { monitoring }\end{array}$ & $\begin{array}{l}8 \text { tests, iv. drug } \\
\text { administration }\end{array}$ & 125 & $\begin{array}{l}29 \text { tests, iv. drug } \\
\text { administration }\end{array}$ & 417 & $292(70)$ \\
\hline \multicolumn{2}{|l|}{ Total } & 14,842 & & 18,612 & $3770(20)$ \\
\hline \multicolumn{6}{|c|}{$\begin{array}{l}\text { † Patients initiated on oral therapy received } 0.0 \text { days iv. and } 47.1 \text { days oral therapy. } \\
\text { Values have been rounded. }\end{array}$} \\
\hline
\end{tabular}

\section{Scenario analyses}

Several scenario analyses were performed to test the effect of uncertainties in the model. As drug acquisition costs for IMD management can vary considerably according to treatment formulation and its effect on administration, two analyses were performed to explore the impact of changing the proportion of ISAV patients initiated on either oral or iv. treatment. In the base case analysis, $75 \%$ of ISAV patients were assumed to be initiated on iv. therapy. In the first scenario analysis, $100 \%$ of ISAV patients were initiated on iv. therapy, before moving to oral therapy in line with the timings in the base case. In the second, 100\% of ISAV patients began treatment on oral therapy. These scenarios were both compared with the base case cost of L-AMB $\rightarrow$ POSA.

A further pair of analyses was performed to explore the impact of variations in the dose of L-AMB [20]. The recommended dose of L-AMB is higher for mucormycosis than for IA $[11,20]$ and, irrespective of suspected infection, clinicians are advised to make dose adjustments to meet individual patient requirements where necessary [20]. Given this potential variation in dose across IMD patients, it was considered useful to test the effect of administering $\mathrm{L}$-AMB at the upper and lower limits of its dosing scale. In the base case, L-AMB was administered at $3 \mathrm{mg} / \mathrm{kg}$ for $50 \%$ of patients and at $5 \mathrm{mg} / \mathrm{kg}$ for the remaining $50 \%$ [20]. In the first scenario analysis, $100 \%$ of L-AMB patients received $3 \mathrm{mg} / \mathrm{kg}$ and in the second, $100 \%$ received $5 \mathrm{mg} / \mathrm{kg}$. These scenarios were both compared with the base case cost of ISAV.

\section{Results}

In the base case analysis, total per patient IMD treatment costs were UK£14,842 with ISAV and UK£18,612 with L-AMB $\rightarrow$ POSA. This equates to a saving of UK£3,770, or 20\%, with ISAV relative to L-AMB $\rightarrow$ POSA. Savings were driven by iv. drug acquisition, and administration $\&$ monitoring costs. Base case results are presented in Table 2 \& Figure 3.

Several scenario analyses were performed to test uncertainty in the model parameters. The first pair of analyses explored the impact of changing the proportion of ISAV patients initiated on either oral or iv. treatment. In both, ISAV remained cost-saving relative to L-AMB $\rightarrow$ POSA:

- When $100 \%$ of ISAV patients were initiated on iv. therapy total treatment costs were UK£15,502 for ISAV compared with UK£18,612 for L-AMB $\rightarrow$ POSA, which represents a saving of UK£3110, or 17\%, with the ISAV regimen (Figure 4).

- When $100 \%$ of ISAV patients were initiated on oral therapy total treatment costs were UK£12,862 for ISAV compared with UK£18,612 for L-AMB $\rightarrow$ POSA, which represents a saving of UK£5750, or 31\%, with the ISAV regimen (Figure 4).

The second pair of scenario analyses was designed to test the effect of varying the dose of L-AMB. Again, ISAV remained cost-saving relative to L-AMB $\rightarrow$ POSA:

- When $100 \%$ of L-AMB $\rightarrow$ POSA patients received L-AMB at a dose of $3 \mathrm{mg} / \mathrm{kg}$ total treatment costs were UK£14,842 for ISAV compared with UK£17,050 for L-AMB $\rightarrow$ POSA, which represents a saving of UK£2209, or $13 \%$, with the ISAV regimen (Figure 5). 


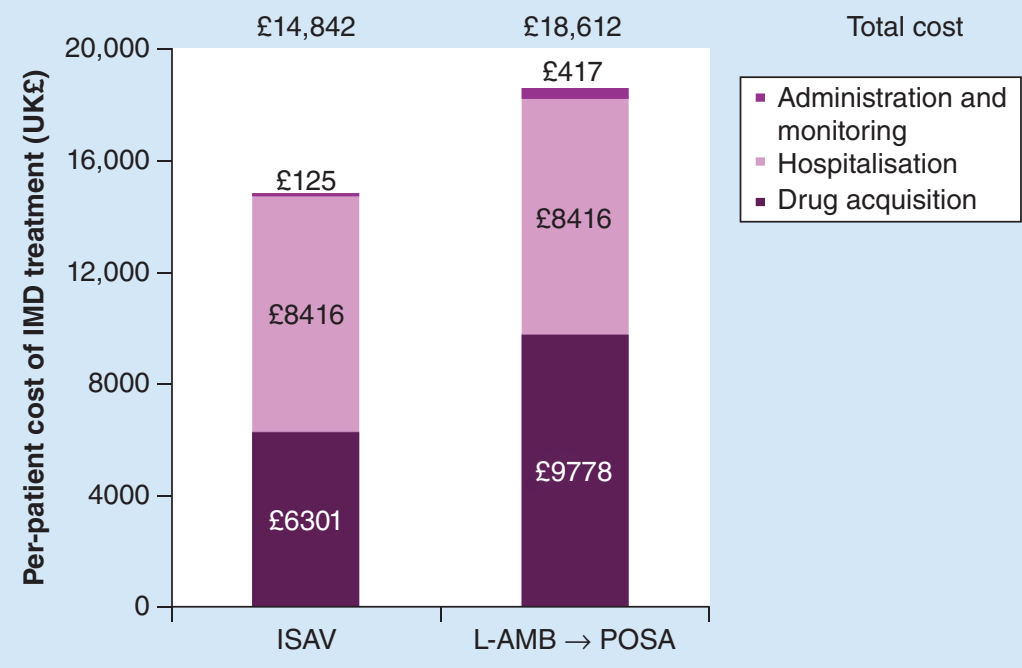

Figure 3. Results of base case cost-minimization analysis. Values have been rounded. IMD: Invasive mold disease; ISAV: Isavuconazole; L-AMB: Liposomal amphotericin B; POSA: Posaconazole.

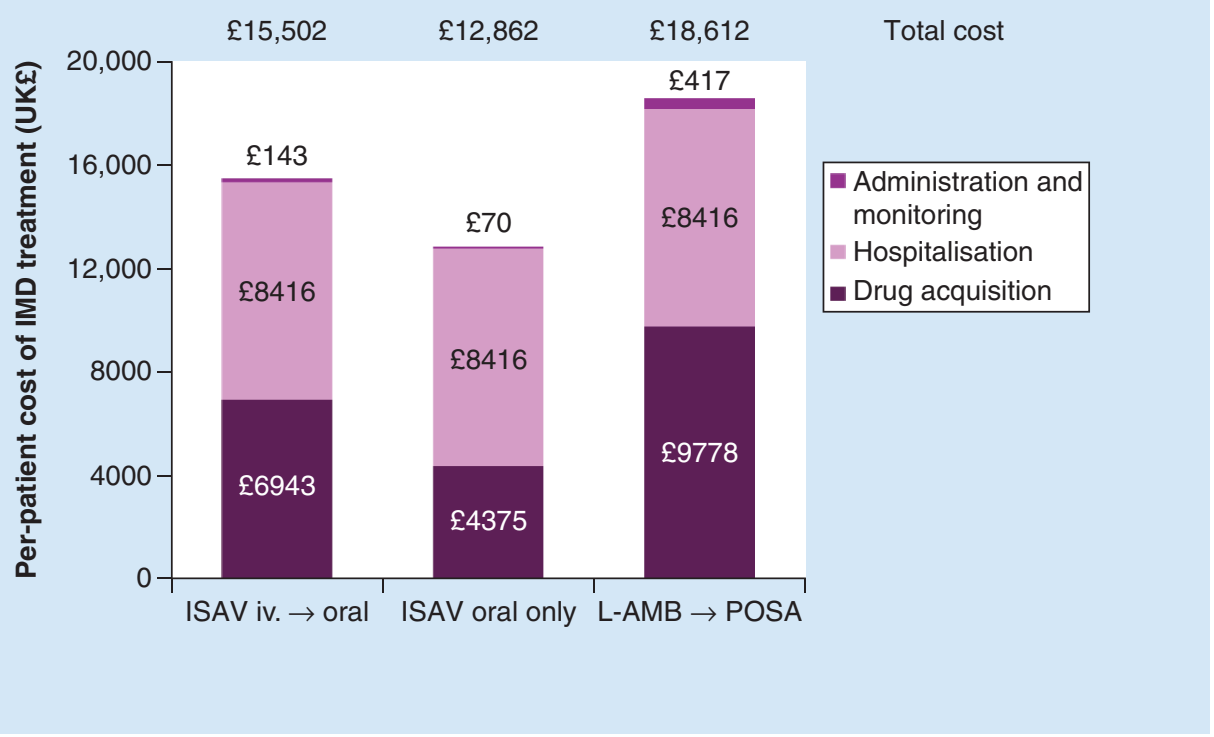

Figure 4. Results of scenario analyses: variation in proportion of isavuconazole patients initiated on intravenous or oral therapy. Values have been rounded.

IMD: Invasive mold disease; ISAV: Isavuconazole; L-AMB: Liposomal amphotericin B; POSA: Posaconazole.

- When $100 \%$ of L-AMB $\rightarrow$ POSA patients received L-AMB at a dose of $5 \mathrm{mg} / \mathrm{kg}$ total treatment costs were UK£14,842 for ISAV compared with UK£20,173 for L-AMB $\rightarrow$ POSA, which represents a saving of UK£5331, or $26 \%$, with the ISAV regimen (Figure 5). 


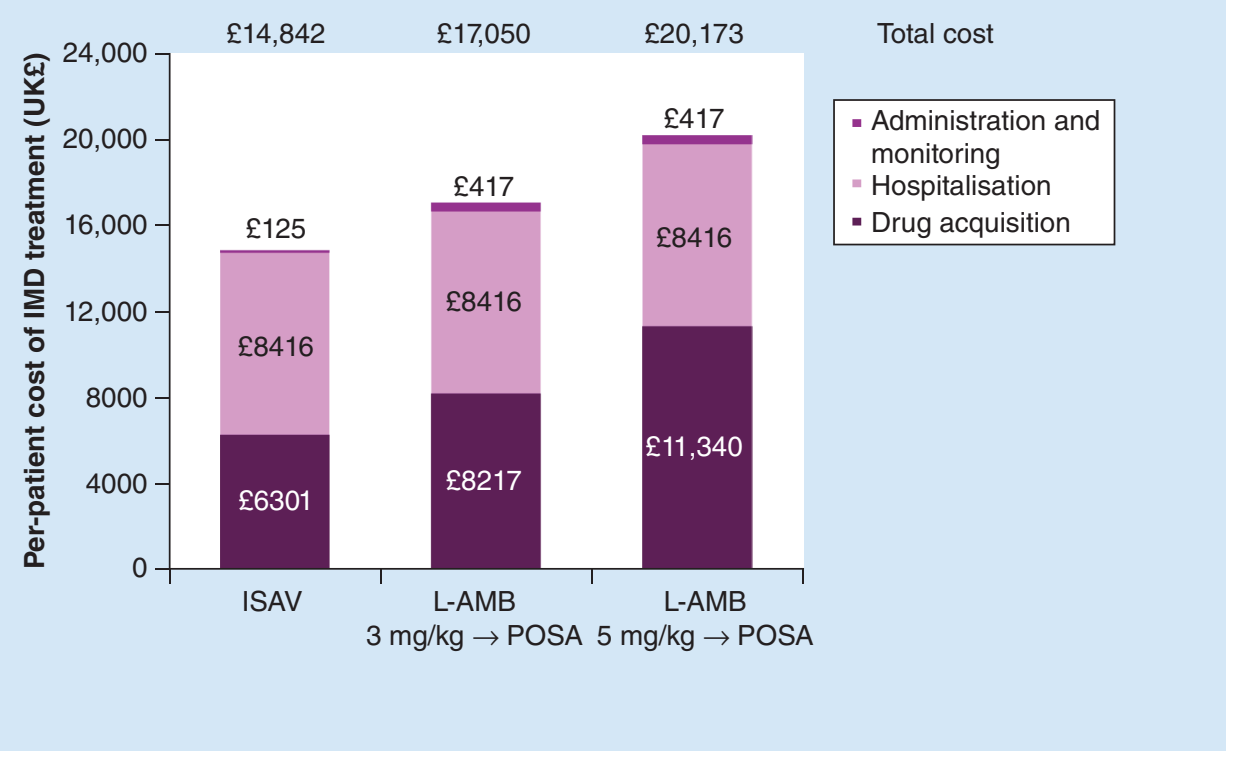

Figure 5. Results of scenario analyses: variation in dose of liposomal amphotericin B. Values have been rounded IMD: Invasive mold disease; ISAV: Isavuconazole; L-AMB: Liposomal amphotericin B; POSA: Posaconazole.

\section{Discussion}

An economic model was developed to estimate the per patient cost of treating IMD patients in the UK with ISAV compared with L-AMB followed by maintenance POSA. The model estimated that use of ISAV was likely to result in substantial cost savings relative to L-AMB $\rightarrow$ POSA. Savings were driven largely by differences in iv. drug acquisition costs, because both daily drug costs and the duration of iv. therapy were lower with ISAV than with L-AMB. Savings were also observed in administration costs - owing to ISAV's shorter iv. infusion preparation time - and monitoring costs, as ISAV required less laboratory testing and TDM than L-AMB $\rightarrow$ POSA.

IMD treatment costs remained lower for ISAV than for L-AMB $\rightarrow$ POSA across scenario analyses in which the proportion of ISAV patients initiated on iv. or oral therapy was varied, and when the dose of L-AMB was set to either $3 \mathrm{mg} / \mathrm{kg}$ or $5 \mathrm{mg} / \mathrm{kg}$, suggesting that the savings observed are likely to be robust to minor adjustments to administration regimens that may occur in clinical practice.

The results of this analysis must be interpreted in the context of several limitations. First, the robustness of the comparison between treatment arms was restricted by the limited availability of data. The underlying assumption of equal clinical outcomes between treatment arms was based on indirect comparison, and resource-use data were taken from different sources for each treatment arm. Further evidence, gathered from real-world experience with ISAV in clinical practice, will be useful to corroborate many of the assumptions made in the model, and may enable a useful update to the analysis to be made in the future.

Another limitation of the analysis was that the L-AMB dose used was higher than that generally recommended for IA patients $(3 \mathrm{mg} / \mathrm{kg})$, who make up a large proportion of the total IMD population. This may have artificially inflated the iv. drug costs for the L-AMB $\rightarrow$ POSA treatment arm. However, the group of IMD patients for whom $\mathrm{L}-\mathrm{AMB}$ is currently the preferred treatment option is likely to include many with a suspicion of mucormycosis, as well as those in whom it can not be excluded, and in these patients, the recommended L-AMB dose is $5 \mathrm{mg} / \mathrm{kg}$. Administering only $3 \mathrm{mg} / \mathrm{kg}$ to patients at risk of mucormycosis may lead to undertreatment. Of note, the recommended dose of ISAV is fixed for all patient groups. In addition, ISAV remained cost-saving in a scenario analysis in which the dose of L-AMB was set to $3 \mathrm{mg} / \mathrm{kg}$ for all L-AMB $\rightarrow$ POSA patients.

The model did not consider differences between treatment arms in the risk of AEs, as the available data sources for each treatment were considered too different to make a robust comparison. Had AEs been included, it is expected that greater savings would have been observed in the ISAV arm, given that ISAV was well-tolerated in the 
SECURE study [17], and the well-documented risk of renal toxicity carried by L-AMB [22-25], which can prolong the duration of hospital stay [6].

Alongside the cost of AEs, several other cost drivers typically seen during IMD treatment were excluded from the model, such as diagnostic imaging and alterations to treatment regimens due to intolerance or refractory disease. However, as there is no evidence to suggest that a difference in these cost drivers exists between treatment arms, it is not expected that their inclusion would have materially changed the outcomes of the model.

Hospitalization costs were also assumed to be equal in both arms, despite differences in the duration of iv. therapy (8.1 days for ISAV vs 14.5 days for L-AMB) that may, in clinical practice, lead to shorter hospital stays in ISAV-treated patients than in those receiving L-AMB. This difference was excluded from the model as many patients with presumed IMD also require hospital care for other reasons. In addition to affecting the duration of hospitalization, the between-treatment difference in the duration of iv. therapy could have other clinical and economic consequences. For example, iv. therapy is a risk factor for healthcare-associated infections [36]. Had this cost driver been included in the model, the relative costs of the L-AMB $\rightarrow$ POSA arm may have increased.

Finally, it should be taken into account that the applicability of the analysis is limited to the current UK NHS perspective. Any generalization beyond these circumstances should be made with caution.

This study is one of the few published economic evaluations of ISAV. A recent cost-minimization analysis, also in the UK NHS setting and based on a similar model structure to the current study, explored the cost of using ISAV for the treatment of mucormycosis, and reported that ISAV would likely be cost-saving relative to L-AMB $\rightarrow$ POSA [37]. Total costs in the mucormycosis analysis, and savings with ISAV relative to L-AMB, were considerably higher than in the current IMD study, owing mainly to the higher dose of L-AMB and prolonged duration of antifungal therapy necessary for successful treatment of mucormycosis [37]. Similarly structured analyses from several other European countries also predict cost savings with ISAV relative to L-AMB $\rightarrow$ POSA in the treatment of both mucormycosis [38,39] and invasive mold disease [40]. Additionally, in IA, a US hospital-perspective analysis of ISAV in comparison with VORI found ISAV to be cost-effective based on lower costs and improved clinical outcomes (deaths avoided and additional clinical responders from SECURE) [41]. These studies lend support to the cost-effectiveness of ISAV but, as clinical practices and payment systems can differ considerably between countries, should be considered in context and the results generalized only with caution.

\section{Conclusion}

IMDs are associated with high morbidity and mortality. Commonly used treatments, including VORI and L-AMB, have several limitations, such as VORI's lack of activity against Mucorales species, and its high risk of AEs and drug-drug interactions, as well as L-AMB's risk of renal toxicity and the need for iv. administration. ISAV has comparable efficacy to standard treatments and an acceptable safety profile. The results of this cost-minimization analysis suggest that use of ISAV in the treatment of IMDs may lead to considerable cost savings relative to a regimen of L-AMB followed by POSA. Further health-economic evaluation is needed to provide confirmation.

\section{Acknowledgements}

E Bagshaw and M Blackney are employees of Covance Market Access, which was commissioned by Basilea Pharmaceutica International Ltd. to provide support with model development and the writing of this article.

Financial \& competing interests disclosure

This study was supported by Basilea Pharmaceutica International Ltd, Basel. D Kuessner and J Posthumus are employees of Basilea Pharmaceutica International Ltd and hold stock options with Basilea Pharmaceutica Ltd. DA Enoch has received funding from Astellas and MSD for conference attendance. The authors have no other relevant affiliations or financial involvement with any organization or entity with a financial interest in or financial conflict with the subject matter or materials discussed in the manuscript apart from those disclosed.

Ethical conduct of research

The authors state that they have obtained research and ethical approval from Mazandaran University of Medical Sciences in all animal experimental investigations. The authors state that they have obtained appropriate institutional review board approval or have followed the principles outlined in the Declaration of Helsinki for all human or animal experimental investigations. In addition, for investigations involving human subjects, informed consent has been obtained from the participants involved. 


\section{Summary points}

- Invasive mold diseases (IMDs) are severe fungal infections, with a high risk of mortality, which affect mainly immunocompromised and critically ill people.

- The cost of treating IMD patients is substantial, largely owing to prolonged use of high-cost antifungals and lengthy hospital stays.

- Voriconazole (VORI) is currently the standard first-line treatment for IMD in the UK as it is relatively inexpensive; however, it lacks Mucorales activity and carries a risk of skin, eye and liver adverse events.

- Liposomal amphotericin B (L-AMB), currently the most commonly used alternative, has broad-spectrum activity, but is associated with renal toxicity and is only available for intravenous administration, so posaconazole (POSA) is often given for maintenance therapy.

- Isavuconazole (ISAV) is a new IMD treatment, indicated in adults for the treatment of invasive aspergillosis and for mucormycosis in patients for whom AMB is inappropriate.

- A cost-minimization model was developed from the perspective of the UK National Health Service to explore the per patient cost (drug acquisition, administration \& monitoring and hospitalization costs) of treating IMD with ISAV versus L-AMB followed by POSA.

- Total per patient treatment costs were estimated to be UK£14,842 with ISAV versus UK£18,612 with L-AMB $\rightarrow$ POSA.

- Savings were primarily driven by lower drug acquisition costs with ISAV relative to L-AMB, alongside lower administration \& monitoring costs.

- ISAV remained cost-saving across various scenario analyses designed to test the model parameters with most uncertainty.

- ISAV appears to be a cost-saving IMD treatment option relative to L-AMB $\rightarrow$ POSA; however, further health-economic evaluations are warranted to confirm this.

\section{Open access}

This work is licensed under the Attribution-NonCommercial-NoDerivatives 4.0 Unported License. To view a copy of this license, visit http://creativecommons.org/licenses/by-nc-nd/4.0/

\section{References}

Papers of special note have been highlighted as: $\bullet$ of interest; $\bullet \bullet$ of considerable interest

1. European Medicines Agency. Committee for Orphan Medicinal Products. Public summary of opinion on orphan designation. Isavuconazonium sulfate for the treatment of invasive aspergillosis (2014).

www.ema.europa.eu/docs/en_GB/document_library/Orphan_designation/2014/07/WC500169890.pdf

2. Bitar D, Lortholary O, Le Strat Y et al. Population-based analysis of invasive fungal infections, France, 2011-2010. Emerg Infect Dis. 20(7), 1149-1155 (2014).

3. European Medicines Agency. Committee for Orphan Medicinal Products. Public summary of opinion on orphan designation. Isavuconazonium sulfate for the treatment of mucormycosis

(2014). www.ema.europa.eu/docs/en_GB/document_library/Orphan_designation/2014/07/WC500169714.pdf

4. Ceesay MM, Sadique Z, Harris R, Ehrlich A, Adams EJ, Pagliuca A. Prospective evaluation of the cost of diagnosis and treatment of invasive fungal disease in a cohort of adult haematology patients in the UK. J. Antimicrob. Chemother. 70(4), 1175-1181 (2015).

- Ceesay 2015 - prospective observational study of the cost of diagnosis and treatment of invasive fungal disease in haematology patients in the UK.

5. Zilberberg MD, Shorr AF, Huang H, Chaudhari P, Federico Paly V, Menzin J. Hospital days, hospitalization costs, and inpatient mortality among patients with mucormycosis: a retrospective analysis of US hospital discharge data. BMC Infect. Dis. 14, 310 (2014).

6. Bates DW, Su L, Yu DT et al. Mortality and costs of acute renal failure associated with amphotericin B therapy. Clin. Infect. Dis. 32(5), 686-693 (2001).

7. Patterson TF, Thompson GR, 3rd, Denning DW et al. Practice guidelines for the diagnosis and management of aspergillosis: 2016 update by the Infectious Diseases Society of America. Clin. Infect. Dis. 63(4), e1-e60 (2016).

8. Vfend $50 \mathrm{mg}$ and $200 \mathrm{mg}$ film-coated tablets, Vfend $200 \mathrm{mg}$ powder for solution for infusion, Vfend $200 \mathrm{mg}$ powder and solvent for solution for infusion, Vfend $40 \mathrm{mg} / \mathrm{ml}$ powder for oral suspension (voriconazole) summary of product characteristics. Pfizer Ltd (2017). www.medicines.org.uk/emc/medicine/10059

9. AmBisome $50 \mathrm{mg}$ powder for solution for infusion (liposomal amphotericin B). Summary of product characteristics. Gilead Sciences Ltd. (2017). www.medicines.org.uk/emc/medicine/1236 
10. Enoch DA, Aliyu SH, Sule O, Lewis SJ, Karas JA. Posaconazole for the treatment of mucormycosis. Int. J. Antimicrob. Agents 38(6), 465-473 (2011).

11. Tissot F, Agrawal S, Pagano L et al. ECIL-6 guidelines for the treatment of invasive candidiasis, aspergillosis and mucormycosis in leukemia and hematopoietic stem cell transplant patients. Haematologica 102(3), 433-444 (2017).

12. Noxafil $100 \mathrm{mg}$ gastro-resistant tablets (posaconazole). Summary of product characteristics. Merck Sharp \& Dohme Ltd (2017). www.medicines.org.uk/emc/medicine/28880

13. Cancidas $50 \mathrm{mg}$ powder for solution for infusion (caspofungin). Summary of product characteristics. Merck Sharp \& Dohme Ltd (2016). www.medicines.org.uk/emc/medicine/12843

14. Viscoli C, Herbrecht R, Akan H et al. An EORTC Phase II study of caspofungin as first-line therapy of invasive aspergillosis in haematological patients. J. Antimicrob. Chemother. 64(6), 1274-1281 (2009).

15. Micallef C, Aliyu SH, Santos R, Brown NM, Rosembert D, Enoch DA. Introduction of an antifungal stewardship programme targeting high-cost antifungals at a tertiary hospital in Cambridge, England. J. Antimicrob. Chemother. 70(6), 1908-1911 (2015).

16. Cresemba $200 \mathrm{mg}$ powder for concentrate for solution for infusion. Summary of product characteristics. Basilea Pharmaceutica International Ltd (2017). www.medicines.org.uk/emc/medicine/31236

17. Maertens JA, Raad II, Patterson TF et al. Isavuconazole versus voriconazole for primary treatment of invasive mould disease caused by Aspergillus and other filamentous fungi (SECURE): a Phase III, randomised-controlled, non-inferiority trial. Lancet 387(10020), 760-769 (2016).

-• Maertens 2016 - Phase III SECURE study of isavuconazole in comparison with voriconazole for the primary treatment of invasive mold disease caused by Aspergillus species and other filamentous fungi.

18. Leenders A, Daenen S, Jansen R et al. Liposomal amphotericin B compared with amphotericin B deoxycholate in the treatment of documented and suspected neturopenia-associated invasive fungal infections. Br. J. Haematol. 103(1), 205-212 (1998).

19. Cornely OA, Maertens J, Bresnik M et al. Efficacy outcomes in a randomised trial of liposomal amphotericin B based on revised EORTC/MSG 2008 definitions of invasive mould disease. Mycoses 54(5), e449-e455 (2011).

20. Marty FM, Ostrosky-Zeichner L, Cornely OA et al. Isavuconazole treatment for mucormycosis: a single-arm open-label trial and case-control analysis. Lancet Infect. Dis. 16(7), 828-837 (2016).

-• Marty 2016 - Phase III single-arm VITAL study of isavuconazole and matched case-control analysis in comparison with liposomal amphotericin $B$ for the primary treatment of mucormycosis.

21. Keirns J, Desai A, Kowalski D et al. QT interval shortening with savuconazole: in vitro and in vivo effects on cardiac repolarization. Clin. Pharmacol. Ther. 101(6), 782-790 (2017).

22. Fleming RV, Kantarjian HM, Husni R et al. Comparison of amphotericin B lipid complex (ABLC) vs ambisome in the treatment of suspected or documented fungal infections in patients with leukemia. Leuk. Lymphoma 40(5-6), 511-520 (2001).

23. Cornely $\mathrm{OA}$, Maertens J, Bresnik $\mathrm{M}$ et al. Liposomal amphotericin B as initial therapy for invasive mold infection: a randomized trial comparing a high-loading dose regimen with standard dosing (AmBiLoad trial). Clin. Infect. Dis. 44(10), 1289-1297 (2007).

24. Lanternier F, Poiree S, Elie C et al. Prospective pilot study of high-dose (10 mg/kg/day) liposomal amphotericin B (L-AMB) for the initial treatment of mucormycosis. J. Antimicrob. Chemother. 70(11), 3116-3123 (2015).

25. Walsh TJ, Pappas P, Winston DJ et al. Voriconazole compared with liposomal amphotericin B for empirical antifungal therapy in patients with neutropenia and persistent fever. N. Engl. J. Med. 346(4), 225-234 (2002).

26. Herbrecht R, Kuessner D, Pooley N, Posthumus J, Escrig E. Meta-analysis of clinical outcomes associated with isavuconazole versus relative comparators for patients with invasive aspergillosis. Presented at: 43rd Annual Meeting of the European Society for Blood and Bone Marrow Transplantation, Marseille, France, 26-29 March 2017.

27. Inflation.eu Worldwide Inflation Data. Inflation Great Britain 2017 (2017). http://inflation.eu/inflation-rates/great-britain/historic-inflation/cpi-inflation-great-britain-2017.aspx

28. Horn D, Goff D, Khandelwal $\mathrm{N}$ et al. Hospital resource use of patients receiving isavuconazole vs voriconazole for invasive mold infections in the Phase III SECURE trial. J. Med. Econ. 19(7), 728-734 (2016).

29. British National Formulary. Isavuconazole (2018). https://bnf.nice.org.uk/medicinal-forms/isavuconazole.html

30. British National Formulary. Amphotericin (2018). https://bnf.nice.org.uk/medicinal-forms/amphotericin.html

31. British National Formulary. Posaconazole (2018). https://bnf.nice.org.uk/medicinal-forms/posaconazole.html

32. Personal Social Services Research Unit. Unit costs of health and social care 2015 (2016). www.pssru.ac.uk/project-pages/unit-costs/unit-costs-2016/

33. NHS reference costs 2015-16. Main schedule (2016). www.gov.uk/government/publications/nhs-reference-costs-2015-to-2016

34. Mycology Reference Centre. Manchester. Antifungal assays (2018). www.mycologymanchester.org/antifungal.php

35. Ashbee HR, Barnes RA, Johnson EM, Richardson MD, Gorton R, Hope WW. Therapeutic drug monitoring (TDM) of antifungal agents: guidelines from the British Society for Medical Mycology. J. Antimicrob. Chemother. 69(5), 1162-1176 (2014). 
36. Cardoso T, Almeida M, Friedman ND et al. Classification of healthcare-associated infection: a systematic review 10 years after the first proposal. BMC Med. 12(40), doi:10.1186/1741-7015-1112-1184 (2014).

37. Bagshaw E, Kuessner D, Posthumus J et al. The cost of treating mucormycosis with isavuconazole compared with standard therapy in the UK. Future Microbiol. 12, 515-525 (2017).

•• Bagshaw 2017 - UK-based cost-minimization analysis between ISAV and L-AMB $\rightarrow$ POSA in the treatment of mucormycosis.

38. Bagshaw E, Carotti A, Blackney M, Chiarini V, Posthumus J, Kuessner D. The cost of treating mucormycosis with isavuconazole compared with liposomal amphotericin B followed by posaconazole in Italy: economic evaluation of the Phase III vital study and fungiscope matched case-control analysis. Presented at: 43rd Annual Meeting of the European Society for Blood and Marrow Transplantation, Marseille, France, 26-29 March 2017.

39. Bagshaw E, Blackney M, Heimann SM et al. The cost of treating mucormycosis with isavuconazole compared with liposomal amphotericin B followed by posaconazole in Germany: economic evaluation of the Phase III VITAL study and FungiScope matched case-control analysis. Presented at: 27th European Congress of Clinical Microbiology and Infectious Diseases, Vienna, Austria, 22-25 April 2017.

40. Bagshaw E, Salaun B, Blackney M, Posthumus J, Kuessner D. The cost of treating invasive mould diseases with isavuconazole compared with liposomal amphotericin B followed by posaconazole in France. Presented at: 28th European Congress of Clinical Microbiology and Infectious Diseases, Madrid, Spain, 21-24 April 2018.

41. Harrington R, Lee E, Yang $\mathrm{H}$ et al. Cost-effectiveness analysis of isavuconazole vs voriconazole as first-line treatment for invasive aspergillosis. Adv. Ther. 34(1), 207-220 (2017). 
